\title{
Une histoire nationale et locale des films fixes d'enseignement
}

\section{Didier Nourrisson}

\section{(2) OpenEdition \\ Journals}

Electronic version

URL: http://journals.openedition.org/trema/3127

DOI: 10.4000/trema.3127

ISSN: 2107-0997

\section{Publisher}

Faculté d'Éducation de l'université de Montpellier

\section{Printed version}

Date of publication: 1 June 2014

Number of pages: 24 - 35

ISBN: 1167-315X

ISSN: 1167-315X

\section{Electronic reference}

Didier Nourrisson, «Une histoire nationale et locale des films fixes d'enseignement », Tréma [Online],

41 | 2014, Online since 01 June 2016, connection on 20 April 2019. URL : http://

journals.openedition.org/trema/3127 ; DOI : 10.4000/trema.3127

This text was automatically generated on 20 April 2019.

Trema 


\title{
Une histoire nationale et locale des films fixes d'enseignement
}

\author{
Didier Nourrisson
}

1 Entre la vue sur verre, projetée par lanterne lumineuse et la diapositive à côté, et quelques fois en concurrence du cinéma, le film fixe tient une place essentielle dans l'art d'enseigner à l'école élémentaire et dans le cycle secondaire durant la période 1925-1975. Support illustratif de la leçon, outil de l'apprentissage, il est devenu moyen pédagogique dans toutes les disciplines du savoir enseigné. Cette place, la première, il l'a conquise au prix de grands débats dans les revues spécialisées et dans les colloques sur l'innovation pédagogique.

2 Le Centre de Recherches, d'Études et de Documentation sur l'Image en Éducation (CREDIE) a retrouvé la plupart de ces films, portant sur toutes les disciplines (histoire, géographie, sciences naturelles, technologie, art, littérature, etc.) et est parvenu à constituer un catalogue informatisé de ces bobines, ouvrant le champ à des recherches multiples et durables.

Découverte fortuitement dans les anciens bains-douches d'une école primaire de la ville de Saint-Étienne, alors qu'était engagée une recherche sur le patrimoine des anciennes écoles normales (équipe Gaspard de l'ancien INRP), une collection disloquée de films fixes d'enseignement s'est véritablement révélée en $1998^{1}$.

Ces films proviennent vraisemblablement de l'ancien Office du cinéma éducateur de la ville de Saint-Étienne. Ils auraient été confiés au CDDP (dans quelles circonstances ?) et déposés là vers 1975, avec des collections de manuels scolaires anciens, dans une recherche d'espace. Mais l'inquiétude grandissante face à la présence de films jugés « inflammables» (une vieille crainte récidivante depuis le grand incendie du Bazar de la Charité) a nécessité leur transfert et surtout l'aiguillon de la recherche sur les anciens procédés d'enseignement justifie leur redécouverte et leur conservation au sein du CREDIE (alors baptisé IREGH).

5 L'histoire de ce procédé pédagogique complètement oublié a pu commencer. En voici les étapes nationales et locales. 
6 Le cinéma s'est rapidement construit comme le $7 \mathrm{e} \operatorname{art}^{2}$. Le mot art doit cependant être pris dans son double sens: une esthétique certes, mais aussi une pratique. Le cinéma reçoit vite en effet une (inj)onction d'efficacité : il doit apprendre autant qu'amuser.

7 À peine le cinématographe est-il né que l'enseignement s'y intéresse : dès 1896, est créé au Musée pédagogique le Service central des projections lumineuses avec une section des vues fixes ${ }^{3}$. Sa mission consiste à faire circuler entre les écoles des collections de vues permettant d'illustrer par des projections les classes et les conférences postscolaires, très en vogue alors. De 54 prêts l'année de création du service, on passe à 44000 au cours de l'année scolaire 1927-1928 ${ }^{4}$. En ce qui concerne les images fixes, il s'agit alors de "vues sur verre », que l'on passe l'une après l'autre avec une lanterne magique; ces vues sur verre (format $8,5 \times 10 \mathrm{~cm}$ ) contribuent à « l'enseignement par l'aspect » que donnent les instituteurs tant à des fins scolaires que pour des cours d'adultes ou des conférences ${ }^{5}$. Mais elles sont d'un coût relativement élevé et cassent facilement.

Durant la première Guerre mondiale, qui met à son tour en valeur la fonction pédagogique et propagandiste du cinéma (cinéma aux armées ${ }^{6}$ ), le pouvoir installe une Commission extra-parlementaire "chargée de rechercher les meilleurs moyens de généraliser l'utilisation cinématographique dans les différentes branches de l'enseignement». Composée de parlementaires, de directeurs et d'inspecteurs de l'enseignement, de professeurs et d'industriels, (67 membres), la Commission rend un rapport favorable en 1920 en ces termes : « à l'heure même où la paix ouvre à notre patrie de nouvelles destinées, il importe de recréer par l'éducation les forces intellectuelles et morales que la guerre a détruites en plein épanouissement. A des besoins nouveaux doivent correspondre des organismes nouveaux. Vous avez eu, Messieurs, la claire vision de ce que l'on peut attendre d'un enseignement rajeuni par l'image et rapproché de la vie. Notre longue enquête aura servi à détruire des préventions et des timidités; elle est de nature à convaincre la Commission du Budget à la Chambre des députés et la Commission des Finances du Sénat et le Ministre de l'Instruction publique qu'il faut sans retard propager l'emploi du cinématographe dans l'enseignement à tous les degrés ${ }^{7}$ ». L'intention est là.

Dans le même temps, la technique s'affine. La société Pathé-Cinéma lance en 1923 un appareil révolutionnaire, le Pathéorama, qui permet de remplacer la vue sur verre, "d'usage général, mais de prix élevé et de manipulation délicate en raison de sa fragilité ${ }^{8}$ " par le film fixe. Ce film, - on parle alors de "photogramme »-, se présente en un rouleau de pellicule photographique solide, large de $35 \mathrm{~mm}$, à double perforation, long parfois d'un mètre. Sur ce support sont réunies entre une douzaine et une centaine d'images, indépendantes les unes des autres, que l'on peut faire défiler à l'écran. En cela il est semblable au film photographique, mais à la différence de ce dernier, le film fixe comporte des vues "positives» (et non négatives comme c'est le cas pour les pellicules photographiques) en noir et blanc (en couleurs après 1960). Les films fixes se rangent aisément dans des petites boites de carton de format parallélépipédique $(4 \times 3 \times 3 \mathrm{~cm})$ ou cylindrique $(4 \times 3 \mathrm{~cm})$; plus tard, les boites seront en bakélite ou en métal. Des maisons d'éditions de manuels scolaires,- Larousse, Nathan - et des entreprises spécialisées Éditions Nouvelles pour l'Enseignement, Office Scolaire de l'Enseignement par le Film - se lancent dans la production.

10 Les exhortations officielles et les avancées techniques ne suffisent pourtant pas. Il faut trouver le moyen de diffuser les films fixes. Dans les années 1920, certaines municipalités encouragent la diffusion sur place de ce matériel éducatif et mettent en place des offices 
du cinéma éducateur : Strasbourg (1920), Lyon et Marseille (1921), Saint-Étienne (1922), Nancy (1923), Paris (1925). Eugène Cauvin (1886-1951), qui faisait déjà avant-guerre des conférences antialcooliques illustrées, bien soutenu par Édouard Herriot, fonde l'Office du Cinéma Éducateur de Lyon': Service municipal en 1921, le voilà proclamé office départemental en 1924. Cauvin estime d'emblée qu'il faut favoriser l'enseignement public et que «mieux vaut préserver aux Offices, et donc à des spécialistes, l'usage de l'outil cinématographique, plutôt que de le confier, sous prétexte de sécurité d'emploi à des enseignants profanes... et éventuellement cinéphiles. » En fait, ces «spécialistes » sont surtout des militants laïcs qui craignent les « profanes » catholiques, groupés autour de la Maison de la Bonne Presse ${ }^{10}$. Les œuvres post-scolaires (sport, art et bien sûr cinéma) sont un puissant moyen pour installer partout les lois laïques et la concurrence accélère les initiatives. Cauvin trouve à Saint-Étienne, cette autre capitale du cinéma des premiers temps ${ }^{11}$, de bons relais avec l'instituteur Eugène Reboul et l'inspecteur d'académie Henri Matte. La municipalité stéphanoise va même jusqu'à doter systématiquement chaque école d'un projecteur Phébus $35 \mathrm{~mm}^{12}$. En effet la plupart des films projetés sont alors des documentaires animés. Beaucoup datent d'ailleurs d'avant 1914 ${ }^{13}$. Les moyens sont d'ailleurs dérisoires pour enrichir le catalogue ou équiper les écoles ${ }^{14}$. Et il faut toute l'énergie de quelques pionniers, comme Gustave Cauvin à Lyon ${ }^{15}$ ou Eugène Reboul à Saint-Étienne, pour faire tourner leurs "filmathèques pédagogiques", comme on les appelle. En 1930, 10000 à 12000 établissements publics, 6000 à 8000 institutions privées disposent d'un appareil cinématographique. Malgré une tentative de fédéralisation des Offices du Cinéma éducateur et un premier (et unique) Congrès à Paris du 26 au 30 septembre 1931, le cinéma d'enseignement tarde à s'imposer.

11 De plus, le film fixe d'enseignement peine indiscutablement à trouver sa place comme outil pédagogique entre le manuel scolaire et le cinéma. Les revues pédagogiques débattent longuement de son utilité ${ }^{16}$. L'inspecteur d'académie de la Loire Henri Matte a pourtant bien vu: "ce sont de véritables leçons où le film qui se déroule, fréquemment fixé, est commenté par le maître, film sur lequel les élèves sont interrogés à chaque instant, interrogations suivies d'un devoir, parfois même d'un croquis. Ce n'est pas un spectacle, une distraction, comme d'aucuns pourraient le croire... ou seraient tentés de le faire! Ce n'est pas non plus une séance hors classe où les élèves de divisions différentes sont réunis dans une salle spéciale de projection, comme pour une représentation " (3 août 1922). Avec le film fixe, l'enseignant a trouvé le moyen privilégié de " provoquer la réflexion, aider à la relation verbale et écrite des faits, préciser les notions données en classe ». L'outil pédagogique est né.

12 Et le système se développe. En 1933 enfin, la Ligue de l'Enseignement crée l'Union française des Offices du Cinéma Educateur Laïque (UFOCEL) qui reprend en main «la tâche de coordonner les efforts de tous les offices et Cinémathèques laïques et de défendre leurs intérêts auprès des pouvoirs publics ${ }^{17}$ ». L'autonomie des Offices n'existe plus; mais la nouvelle organisation fait vite la preuve de son efficacité et de son utilité. Elle est encouragée officiellement. Le 8 octobre 1935, le Ministre de l'Éducation nationale, Mario Roustan, fait savoir à tous les inspecteurs d'Académie que "des subventions pourront être accordées sur les crédits du Ministère de l'Éducation nationale pour l'acquisition, sous réserve de l'approbation technique, d'appareils de projections ${ }^{18} \ldots$... 17 OCE fonctionnent sur le territoire national à la fin des années 1930.

Roustan parle aussi des appareils de projection fixes. Car le triomphe du cinématographe, art du mouvement par définition, n'a pas entrainé la disparition des vues fixes. Certes la lanterne magique et ses vues sur verre, trop fragiles, ne connaissent plus guère 
d'audience après la première Guerre mondiale ${ }^{19}$. Il reste cependant des adeptes du fixe, si l'on en considère le surgissement de nombreux projecteurs-visionneuses dans les années 20 , capable de «lire» image par image, des films standards de $30 \mathrm{~mm}$ en acétate de cellulose ${ }^{20}$.

Certains soulignent à l'envi la complémentarité des deux procédés éducatifs ${ }^{21}$. Et Célestin Freinet comprend l'intérêt du film fixe pour une pédagogie active. Son mouvement s'engage dans la publication de documentation pour l'école. Alfred Carlier, un archiviste ami de Freinet, qui a créé l'office de Documentation historique et archéologique et la Bibliothèque de Travail en 1930, fonde une société d'édition en 1935 et se met à réaliser des films fixes avec les vues tirées de ses brochures. Le travail, tant scientifique que pédagogique, est tout simplement remarquable.

La marche à la guerre tempère sûrement les meilleures dispositions en faveur du cinéma d'éducation, qu'il soit fixe ou animé. Mais les Offices du cinéma éducateur poursuivent avec ténacité leur équipement. À Saint-Étienne, le rapport d'activité du nouveau directeur, Jean-Baptiste Cancade, signale la présence de 335 films fixes dans les collections en 1938 ; ils seront 2135 en 1942-43 ${ }^{22}$.

Après 1945, l'UFOCEL laisse la place à l'UFOLEIS (Union Française des CEuvres Laïques d'Éducation par l'Image et par le Son) et les Offices (un dans chacune des vingt académies) qu'elle fédère s'intitulent désormais OROLEIS (Office Régional des CEuvres Laïques d'Éducation par l'Image et par le Son). Un nouveau souffle anime les éducateurs. Dans ce domaine aussi, c'est la reconstruction. «Le cinéma d'enseignement français est solidaire de la vie de la nation. Par chance et par volonté il a échappé à la maladie générale : il n'existe pas un seul film d'enseignement où la marque de l'ennemi ni celle de ses collaborateurs français ait pu s'imprimer, pas un où leur influence puisse se déceler ${ }^{23}$ ». Une Commission se met en place avec l'arrêté du 1er décembre 1944, dite Commission ministérielle du Cinématographe d'Enseignement, sous la direction de Grandjouan, chef du service de documentation et d'études pédagogiques. Elle reçoit le pouvoir de délivrer un agrément pour les projecteurs et d'attribuer le visa «film d'enseignement et d'éducation ». La liste en est régulièrement publiée dans UFOCEL Informations.

Les films doivent clairement éduquer à la paix : «le bon instituteur sait qu'il peut aider à construire un monde meilleur en développant chez ses élèves le respect de la personnalité humaine, en leur faisant comprendre que la prospérité des autres peuples dans toutes les parties du monde, se répercute sur leur propre bien-être, et en les menant à désirer que les droits fondamentaux de l'homme soient étendus à tous les peuples du monde ${ }^{24} »$.

Les films fixes, désormais dotés comme les autres d'une double ligne de perforations, donc en largeur $35 \mathrm{~mm}$, connaissent un indéniable renouveau. Le nombre des éditeurs se multiplie rapidement, en même temps que les types d'appareils sur le marché. Les éditeurs sont classés selon leur choix confessionnel et la compétition relancée entre « laïcards » et cléricaux dans ses années d'après-guerre pourrait expliquer en partie ce nouvel engouement. Animé de l'esprit de l'encyclique de Pie XII «vigilanti cura» qui prône un redressement moral, le $4 \mathrm{e}$ Congrès international du Cinéma (catholique) se tient à Bruxelles en $1947{ }^{25}$ et lance l'Église "à la conquête du cinéma ${ }^{26}$ ». Le cinéma d'enseignement passe en première ligne comme instrument de moralisation, valeur que se disputent les cléricaux et les « laïcards ». « Il appartient aux laïques d'encourager la production et de soutenir vigoureusement les œuvres cinématographiques de vérité qui aident les masses de spectateurs à se délivrer des antiques complexes, à pénétrer les 
contradictions de notre univers, et les stimuler à créer eux-mêmes les conditions de leur bonheur ${ }^{27}$ ». Par ailleurs, la massification de l'enseignement, lié au célèbre " baby boom ${ }^{28}$ ", exige l'usage de techniques pédagogiques rajeunies et plus actives. Le film, dans la modernité d'une arrivée dans la " vidéosphère ${ }^{29}$ ", trouve toute sa place à l'école après la guerre. Les sujets abordés par les "photogrammes» sont des plus variés. Ils balaient toutes les disciplines enseignées et couvrent tous les programmes: lettre, histoire, géographie, science de la vie et de la terre, sport, sciences physiques, technologies... De nombreux films traitent aussi de sujets transversaux (santé, hygiène, actualités, catéchisme, orientation professionnelle...). Bon nombre de films sont destinés aussi à la récréation scolaire, au patronage et adoptent un contenu ludique, sans perdre leur intention moralisante. On opposera ainsi la série laïque des Sylvain et Sylvette aux catholiques Aventures du Petit Negro.

Les types de films agréés et catalogués ${ }^{30}$

\begin{tabular}{|c|c|c|c|c|}
\hline Types & 1947 & 1949 & 1955 & 1959 \\
\hline Histoire & 42 & 290 & 2207 & 2888 \\
\hline Géographie & 9 & 44 & 357 & 382 \\
\hline $\begin{array}{c}\text { Sciences et } \\
\text { Techniques }\end{array}$ & 2 & 67 & 314 & 815 \\
\hline $\begin{array}{c}\text { Sciences } \\
\text { naturelles }\end{array}$ & 1 & 51 & 339 & 357 \\
\hline Arts & 6 & 11 & $?$ & 24 \\
\hline Littérature & 5 & 4 & 364 & 595 \\
\hline Divers & 3 & 37 & 225 & 284 \\
\hline Total & 68 & 504 & 3836 & 5345 \\
\hline
\end{tabular}

L'usage du film fixe ne s'épanouira réellement qu'au début des années cinquante. En 1951 en effet, la loi Barangé accorde aux établissements scolaires une importante dotation pour l'achat de matériel d'enseignement, en particulier pour l'acquisition d'appareils de projections fixes. De plus, avec l'essor économique, les grandes entreprises subventionnent des opérations de promotion des produits industriels ou de services auprès des écoles. Une maison d'édition réalise même les commandes: l'office de Documentation par le Film fabriquent des dizaines de films fixes "offerts à l'enseignement " par les entreprises automobiles (Renault, Citroën), les entreprises de biens ménagers (ElectroLux, Fly Tox, Fédération de la Savonnerie), les industries alimentaires (Fédération des Spiritueux), les banques (Caisse d'Epargne). L'État, par le biais de ses institutions para-publiques (Sécurité Sociale, Comité National de Défense Contre l'Alcoolisme) n'est pas en reste. Les pays étrangers et organisations internationales, parfois gros producteurs eux-mêmes de films fixes, ne manquent pas non 
plus d'inonder le marché scolaire français. On a récemment découvert des collections de films fixes nord-américains (canadiens, états-uniens, ONU) ${ }^{31}$.

Par discipline, la revue des séries de films fixes se décompose ainsi :

Histoire :

Toutes les périodes sont représentées depuis la Préhistoire, car les programmes d'histoire suivent la chronologie. À cela, il faut ajouter plusieurs séries de films d'histoires thématiques (paysans, habitat, transport, aviation...) ; c'est peut-être en lien avec la « nouvelle histoire».

L'étude de la Seconde Guerre mondiale, de la Commune de Paris, a déjà commencé.

Géographie :

Figurent des généralités sur les concepts et sur les thèmes de géographie physique et humaine. Beaucoup de films - à peu près un quart - traitent de la géographie régionale. Nous noterons aussi le formidable intérêt des films sur les colonies françaises, juste avant les indépendances. Là encore, un riche travail sur les représentations commence à être conduit.

\section{Sciences et techniques :}

Beaucoup d'éléments portent sur les métiers et les filières industrielles. Notons quelques films sur les "transmissions modernes de la pensée », y compris la télévision et les satellites (nous ne sommes pourtant qu'en 1959 !).

Sciences de la Vie et de la Terre :

Sans surprise, les films portent sur la physiologie de l'homme, les animaux, les plantes.

De nombreux films ont également trait à l'hygiène : la puériculture, l'hygiène des grandes fonctions (digestion, respiration, vision, ...) ; les maladies « contagieuses » (choléra), et les maladies dites «sociales» (tuberculose). Dans ce dernier domaine, les films traitant de l'alcoolisme forment un solide corpus de 21 films (plus de mille vues). Ces images de l'alcoolisme dans les années 50 mêlent un regard très passéiste sur le phénomène du tropboire (reprise de certaines vues sur verre de la Belle Époque) et des photos récentes (par exemple, celle d'un accident qui aurait été provoqué par l'imbibition du chauffard) ${ }^{32}$.

\section{Littérature :}

On note la présence d'importantes collections de films de distraction: Perlin et Pimpin, Sylvain et Sylvette, Tintin Minet, des contes et histoires du monde. Mais aussi quelques figurations d'œuvres littéraires: fabliaux du Moyen-Âge, et quelques florilèges de la littérature française - Maupassant, Hugo, Balzac - ou étrangère - Cervantes, Andersen.

\section{Divers :}

La collection des films sur le sport s'élève à 85 : techniques sportives, massages, Olympiades (Vienne, 1931 ; Berlin, 1936). Leur étude vient de s'achever ${ }^{33}$.

Dans le domaine de la pédagogie, plusieurs films portent sur l'apprentissage de la lecture, du calcul, ou encore du dessin ${ }^{34}$.

24 On trouve aussi, en ce temps chéri des jolies colonies de vacances, 3 films sur les Éclaireurs de France, deux sur les écoles de plein air. Enfin, sous la rubrique " sociologie ", on est surpris de voir et lire un Plan quinquennal en URSS ${ }^{35}$ ou La guerre chimique, ou encore des considérations démographiques comme « la France ne doit pas être un pays de fils uniques et de célibataires ».

Les collections de films fixes enflent donc singulièrement jusqu'à la fin des années 1960 . C'est le cas en particulier au sein de l'office du cinéma éducateur de la Loire comme du service audio-visuel du diocèse de Saint-Étienne ${ }^{36}$. 
Films fixes de l'Office du Cinéma Éducateur de la Loire (doubles compris).

\begin{tabular}{|l|l|l|l|l|}
\hline 1938 & 1942 & 1948 & 1952 & 1960 \\
\hline 335 & 2135 & 3800 & 8000 & 8951 \\
\hline
\end{tabular}

Ces films ont été utilisés dans et hors les classes durant des décennies. Ils servent de support visuel à l'enseignant(e) lors de séquences d'enseignement diverses et pour tout niveau: du cours préparatoire auquel il faut apprendre à lire à l'école supérieure de technologie, qui rassemble des jeunes en formation continue. Ils sont mis en œuvre aussi lors des séances récréatives de patronage ${ }^{37}$ ou en colonies de vacances. L'instituteur ou le professeur projette et commente l'une après l'autre les diverses vues. Il peut s'aider d'une notice qui accompagne parfois l'envoi du film. Les vues semblent agir comme un " charme » sur l'esprit des élèves, destiné à faire " passer » la parole du maître.

L'image fixe a reçu droit de cité (scolaire) dans les années 1925-1975.En ralentissant le défilement des images - 1 image/minute au lieu de 24 images/seconde -, le film fixe fait du cinéma - par définition écriture du mouvement -, un enseignement pratique, plus qu'une pratique d'enseignement. 7e art pour esthètes, le cinéma s'est trouvé une pratique pédagogique, une conduite de classe. La suite est bien sûr moins glorieuse. Les catalogues, au début des années 1970, se remplissent de séries de diapositives qui effacent assez rapidement les films fixes. L'enseignant trouve dans les diapositives, assez solides désormais pour «tenir » à la chaleur du projecteur, des images qu'il peut agencer dans son cours suivant ses intentions et sa liberté pédagogique. Le film fixe est tombé dans les oubliettes de l'histoire. Cependant, nos modernes powerpoint n'assurent-ils pas une véritable résurgence du film fixe, la souplesse d'utilisation en plus?

Film fixe, « Renault à travers l'histoire », Office de documentation par le film [1955]. Cedrhe : 50377.

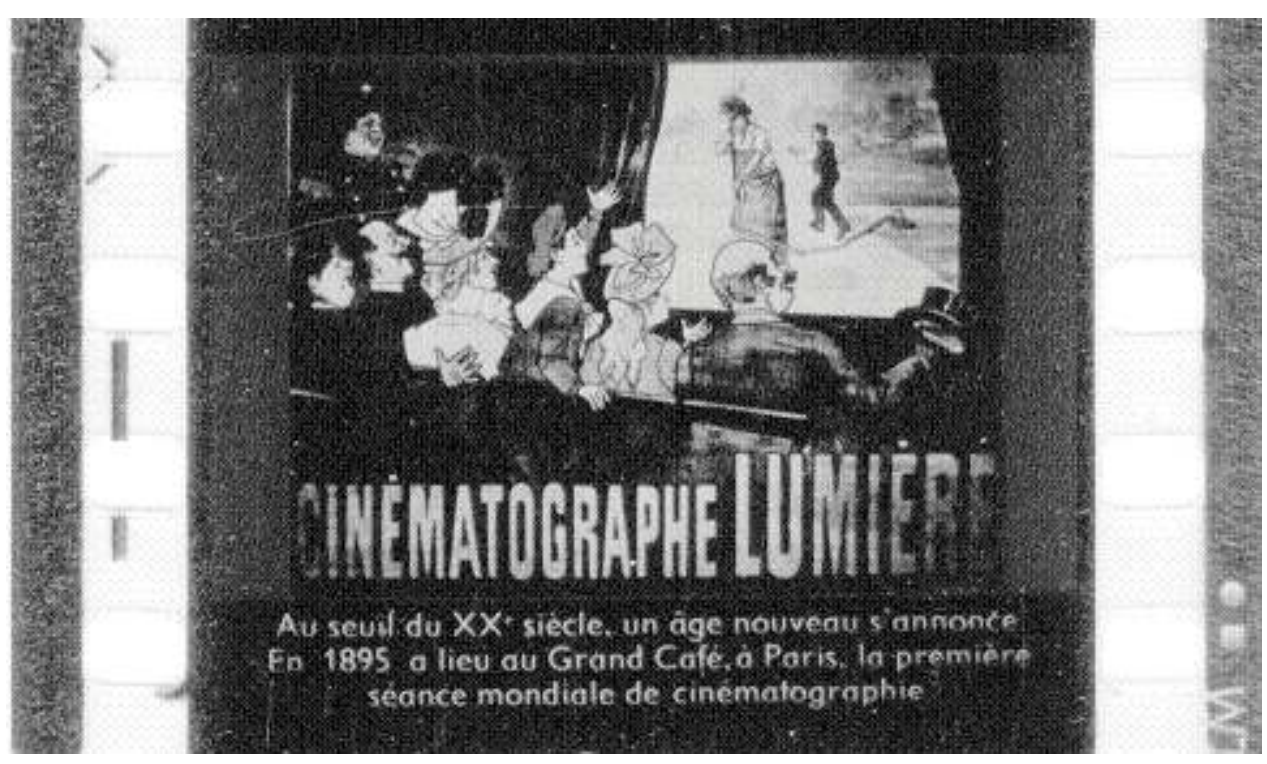




\section{BIBLIOGRAPHY}

Borde, R., Perrin, C. (1992). Les Offices du Cinéma éducateur et la survivance du muet 1925 - 1940. Lyon : Presses Universitaires de Lyon.

Caumeil, J.-G., Charroin, P., Lignon, F., Nourrisson, D. (2010). Fixité de l'image, mobilité des corps. L'enseignement des activités physiques et sportives par le film fixe. Clermont-Ferrand : Impr. Diazo, 200p. + CD Rom.

Freyssinet-Dominjon, J., Nourrisson, D. (2008). L'école face à l'alcool. L'enseignement de l'antialcoolisme en France 1870-1970. Saint-Étienne : Publications de l'Université de SaintÉtienne, coll. IUFM (avec CD Rom).

Nourrisson, D. (2013). Un patrimoine audio-visuel canadien méconnu : les films fixes d'enseignement. Sociétés et Représentations, $\mathrm{n}^{\circ 35}$, Archives et patrimoines visuels et sonores. Nourrisson, D. (2012). Quelles images pour éduquer. In D. Berger, C. Simar (dir.), Éducation à la santé dans et hors de l'école. Paris : MGEN/Unirès.

Nourrisson, D. (2012). Films fixes de l'ONF en France : un certain regard sur le Canada.

Encyclopédie du patrimoine culturel de l'Amérique, art. 301.

En ligne : http://www.ameriquefrancaise.org

Nourrisson, D. (2011). Un fonds éducatif réinventé. Revue Sociétés et Représentations. En ligne : http://www.cairn.info/revue-societes-et-representations-2011-1-p-177.htm

Nourrisson, D. (2011). Les films fixes de santé : des documents pédagogiques riches d'enseignement. In F. Douguet, T. Fillaut, F.-X. Schweyer (dir.), Image et santé. Matériaux, outils, usages. Rennes : Presses de l'EHESP.

Nourrisson, D. (2008). Quand la sécurité sociale faisait la promotion de la santé. Histoire de l'éducation, $n^{\circ} 129$, « La promotion de la santé au travers des images véhiculées par les institutions sanitaires et sociales », actes du colloque sur l'histoire de la protection sociale, Arles, avril 2007. Paris : Association pour l'étude de l'histoire de la Sécurité sociale.

Nourrisson, D. (2008). La Libération enseignée à l'école de la quatrième République in

M., Lebrun ; P. ,Aubin et al. (Dir.), Les manuels d'ici et d'ailleurs, d'hier à demain. Montréal : Presses de l'UQAM, CD Rom.

Nourrisson, D. (2008). Quand l'image fait école. L'exemple de l'antialcoolisme. Revue InterCDI, $\mathrm{n}^{\circ}$ spécial.

Nourrisson, D. (2008). Des films fixes pour enseigner. Revue InterCDI, $n^{\circ} 210$.

Nourrisson, D. (2008). La Loire, un patrimoine éducatif in D. Nourrisson et J.-F. Brun (dir.), La Loire, un territoire en quête d'identité. Saint-Étienne : Publications de l'Université de SaintÉtienne.

Nourrisson, D. (2005). L'image des Russes dans l'enseignement français. In D. Nourrisson et Y. Perrin (dir.), Le barbare, l'étranger : images de l'autre. Saint-Étienne : Publication de l'Université de Saint-Etienne, coll. CERHI. 
Nourrisson, D. (2006). Le faux et la faucille : l'enseignement du réalisme soviétique en France par le film fixe. In M.-C. Baquès, A. Duprat (dir.), Art-Image-Histoire : Le faux et la fiction : Actes du colloque, 23-24 mars 2004. Clermont-Ferrand : CRDP d'Auvergne/Université Blaise Pascal.

Nourrisson, D. (2001). Le 7e art... d'enseigner : le film fixe. In P. Jeunet, D. Nourrisson (dir.), Cinéma-école : aller-retour. Actes du colloque de Saint-Étienne. Novembre 2000. Saint-Étienne : Publications de l'Université de Saint-Étienne.

Pastre-Robert, B., Dubost, M., Massit-Folléa, F. (dir.), Cinéma pédagogique et scientifique. À la redécouverte des archives. Lyon ENS éditions.

Senthiles, A. (1996). L'audiovisuel au service de l'enseignement: projections lumineuses et cinéma scolaire, 1880-1940. La Gazette des archives, $n^{\circ} 173$, 2e trimestre.

Vignaux, V. (2007). Jean Benoit-Lévy ou le corps comme utopie, une histoire du cinéma éducateur dans l'entre-deux-guerres en France. Paris : AFRHC.

Zarch, F. (1996). De l'idée d'image à l'image en mouvement. In D. Nourrisson et A. Vaillant (dir.), Corps en mouvement. Saint-Étienne : Publications de l'Université de Saint-Étienne.

\section{NOTES}

1. Sur l'indication des membres de l'association L'école d'hier à aujourd'hui que nous remercions vivement à nouveau. Notre catalogue informatique contient désormais 9600 références de films fixes. Site web : films-fixes.univ-lyon1.fr

2. L'écrivain italien, Riciotto Canudo, publie le 28 mars 1911 son Manifeste des sept arts. Il y distingue les arts de l'espace (architecture, sculpture, peinture) et les arts du temps (musique, poésie, danse) et fait du cinéma un art de synthèse.

3. Le Musée pédagogique a été fondé en 1879 par Ferdinand Buisson, alors directeur de l'enseignement primaire. Hormis le Musée pédagogique, le Ministère de l'Agriculture, la Direction de l'enseignement technique et le Ministère de l'hygiène fournissent des films pédagogiques (animés seulement, semble-t-il)

4. Senthiles, A. (1996). L'audiovisuel au service de l'enseignement: projections lumineuses et cinéma scolaire, 1880-1940. La Gazette des archives, $n^{\circ} 173$, 2e trimestre, pp.176-180.

5. Cf. Meunier, S. (1880). Les projections lumineuses et l'enseignement primaire. Paris : Molteni, A. ; Clerc, H., «Projections lumineuses» dans le Dictionnaire de Pédagogie et d'Instruction primaire de Ferdinand Buisson (1887 et 1911), ou encore Les projections lumineuses à l'école, aux cours du soir et en famille, de René Leblanc (1904). Cf. aussi la revue de pédagogie Après l'école, à partir de 1895.

6. En 1920, Pierre Marcel, ancien chef de la Section photographique et cinématographique de l'Armée, est le premier à établir, pour le compte de la Compagnie universelle cinématographique, le répertoire de tous les films, tant en France qu'à l'étranger, pouvant servir à l'enseignement.

7. Cité in Lang, A. (1948). Le tableau blanc. Paris : Horizons de France, p.128.

8. Pathéorama (Notice technique), Paris, Pathé-Cinéma, (s.d.).

9. Gustave Cauvin est un militant anarchiste, laïcard, haut en couleurs, qui n'hésite pas à s'engager avec fougue. Cf. Nourrisson, D. (1997). Une tournée antialcoolique pendant la grande Guerre. Cahiers d'histoire, tome XLII, n¹, pp.109-121.

10. La Maison de la Bonne Presse réalise des films par dizaines dans l'entre-deux-guerres pour une éducation religieuse (série vie de Jésus, Histoire des saints, Histoire de l'Église) et une distraction morale (bande dessinée Le petit Negro,...). Elle vend aussi des projecteurs sous sa marque. 
11. Zarch, F. (2000). Catalogue des films projetés à Saint-Étienne avant la première guerre mondiale. Saint-Étienne : Publications de l'Université de Saint-Étienne.

12. Cf. Ravat, A. (1996). L'Office du cinéma éducateur de Saint-Étienne 1922-1952. Mémoire de maîtrise d'Études cinématographiques et audiovisuelles, sous la dir. de Jacques Gerstenkorn, Université Lyon II.

13. Delmeulle, F. (1996). Gaumont et la naissance du cinéma d'enseignement 1909-1914. In M. Marie (dir.), Les vingt premières années du cinéma français. Paris: Presses de la Sorbonne nouvelle, pp.67-75, indique que les films que regroupe le catalogue Gaumont des années 20 proviennent dans une proportion proche de $50 \%$ des années d'avant-guerre.

14. Delmeulle, F. (Cf. supra. p. 75) parle de 250000 francs alloués par le ministère de l'Instruction publique là où il aurait fallu 80 millions de francs pour le seul équipement en matériel des écoles primaires.

15. On doit à Gustave Cauvin des ouvrages et brochures remarquables sur les débuts du cinéma éducateur, comme Le cinéma éducateur à l'école et dans nos œuvres, Lyon, Office du cinéma éducateur, Conseil général de la Ligue de l'enseignement, 1939.

16. Cf. Renonciat, A. (2004). Vue fixe et/ou cinéma dans l'enseignement: naissance d'une polémique (1916-1922). De Pastre, B., Dubost, M., Massit-Folléat, F. (2004). Cinéma pédagogique et scientifique : à la redécouverte des archives. Lyon : ENS Éditions, op.cit., pp. 61-72 ; FreyssinetDominjon, J., Nourrisson, D. (2008). L'école face à l'alcool. L'enseignement de l'antialcoolisme en France 1870-1970. Saint-Étienne : Publications de l'Université de Saint-Étienne, coll. IUFM, op.cit., pp.105-115.

17. Borde, R., Perrin, C. (1992). Les Offices du Cinéma éducateur et la survivance du muet 1925-1940. Lyon : Presses Universitaires de Lyon.

18. André Lang, Le tableau blanc, op. cit., p. 137.

19. Mannoni, L. (1990). Plaque de verre ou celluloïd ? Lanterne magique et cinéma : la guerre d'indépendance. $1895, \mathrm{n}^{\circ} 7$, pp. 3-27.

20. Mannoni, L. (1996). Le mouvement continué. Catalogue illustré de la collection des appareils de la Cinémathèque française. Paris - Milan : Cinémathèque française - Mazzota ; et sur l'un de ces appareils: Lefebvre, T. (1997). Sage comme une image. L'abbé Songaylo et le Pathéorama. 1895, n²1, pp. 147-153.

21. Ueberschlag, J. (2007). Jean Brérault, l'instituteur cinéaste (1898-1973). Saint-Étienne: Publication de l'Université de Saint-Étienne, coll. IUFM.

22. Cf. Ravat, A. (1996). L'Office du cinéma éducateur de Saint-Étienne 1922-1952. Mémoire de maîtrise d'Études cinématographiques et audiovisuelles, sous la dir. de Jacques Gerstenkorn, Université Lyon II.

23. Propos de Jean Brérault, réalisateur de nombreux films scolaires, fin 1946, cités in André Lang, Le tableau blanc, op. cit., p.139.

24. Berg, E.L., Bleich, D.-M. (1950). Le cinéma et la paix. UFOCEL Informations, $n^{\circ} 8$.

25. Après ceux La Haye (1928), Munich (1929), Bruxelles (1935).

26. Selon le titre inquiet de la revue UFOCEL Information $n^{\circ} 13$, de mai 1948, p. 3-4.

27. UFOCEL Informations, déc. 1948, p. 5

28. Selon Prost, A. (1986). L'enseignement en France 1800-1967. Paris : Armand Colin, p. 435. Entre 1945-50 et 1963-64, les effectifs du primaire passent de 5,120 millions à 8,212 et ceux du secondaire de 507000 à 1371000 .

29. Terme emprunté à Régis Debray dans son Cours de médiologie générale.

30. Les catalogues de référence sont ceux de l'Office du Cinéma Éducateur de la Loire publiés à Saint-Étienne et de la Fédération française du Cinéma Éducateur publié à Paris.

31. Cf. Nourrisson, D. (2013). Un patrimoine audio-visuel canadien méconnu: les films fixes d'enseignement. Sociétés et Représentations, n³5, Archives et patrimoines visuels et sonores, 
pp. 77-95 ; Nourrisson, D. (n.d.). Films fixes de l'ONF en France : un certain regard sur le Canada. Encyclopédie du patrimoine culturel de l'Amérique, art. 301.

32. Freyssinet-Dominjon, J., Nourrisson, D. (2008). L'école face à l'alcool. L'enseignement de l'antialcoolisme en France 1870-1970. Saint-Étienne: Publications de l'Université de SaintÉtienne, coll. IUFM.

33. Caumeil, J.-G., Charroin, P., Lignon, F., Nourrisson, D. (2010). Fixité de l'image, mobilité des corps. L'enseignement des activités physiques et sportives par le film fixe. Clermont-Ferrand: Impr. Diazo.

34. On se rappelle que les premiers films d'enseignement (animés), réalisés par Adrien Bruneau dans les années 20, portent sur l'art du dessin.

35. Nourrisson, D. (2005). L'image des Russes dans l'enseignement français. In Nourrisson, D. et Perrin, Y. (dir.), Le barbare, l'étranger: images de l'autre. Saint-Étienne: Publication de l'Université de Saint-Etienne, coll. CERHI, pp. 305-316.

36. Les collections diocésaines (près de 4000 films fixes) viennent d'arriver au CREDIE en plus du fonds initial de l'OCE, des collections d'écoles publiques et privées, et des archives municipales (Arras, Besançon).

37. Dessertine, D., Maradan, B. (2001). L'âge d'or des patronages (1919-1939). La socialisation de l'enfance par les loisirs. Vaucresson : CNFE-PJJ.

\section{ABSTRACTS}

From the filmstrip collection of the Centre de Recherches, d'Études et de Documentation sur l'Image en Education (CREDIE) concerning all the disciplines (history, geography, natural science, technology, art, literature, etc), we have formed a computerized catalogue of these filmstrips, opening up the field of many researches. Through the analysis of this collection we can make a study of the filmstrip.

À partir des fonds de films fixes du Centre de Recherches, d'Études et de Documentation sur l'Image en Éducation (CREDIE) portant sur toutes les disciplines (histoire, géographie, sciences naturelles, technologie, art, littérature, etc.), nous avons pu constituer un catalogue informatisé de ces bobines, ouvrant le champ à des recherches multiples. L'analyse de ce fonds permet d'étudier le film fixe.

\section{INDEX}

Mots-clés: CREDIE, film fixe, histoire de l'éducation

Keywords: CREDIE, education history, filmstrips

\section{AUTHOR}

\section{DIDIER NOURRISSON}

Professeur d'histoire contemporaine, ESPE/CREDIE de Lyon 